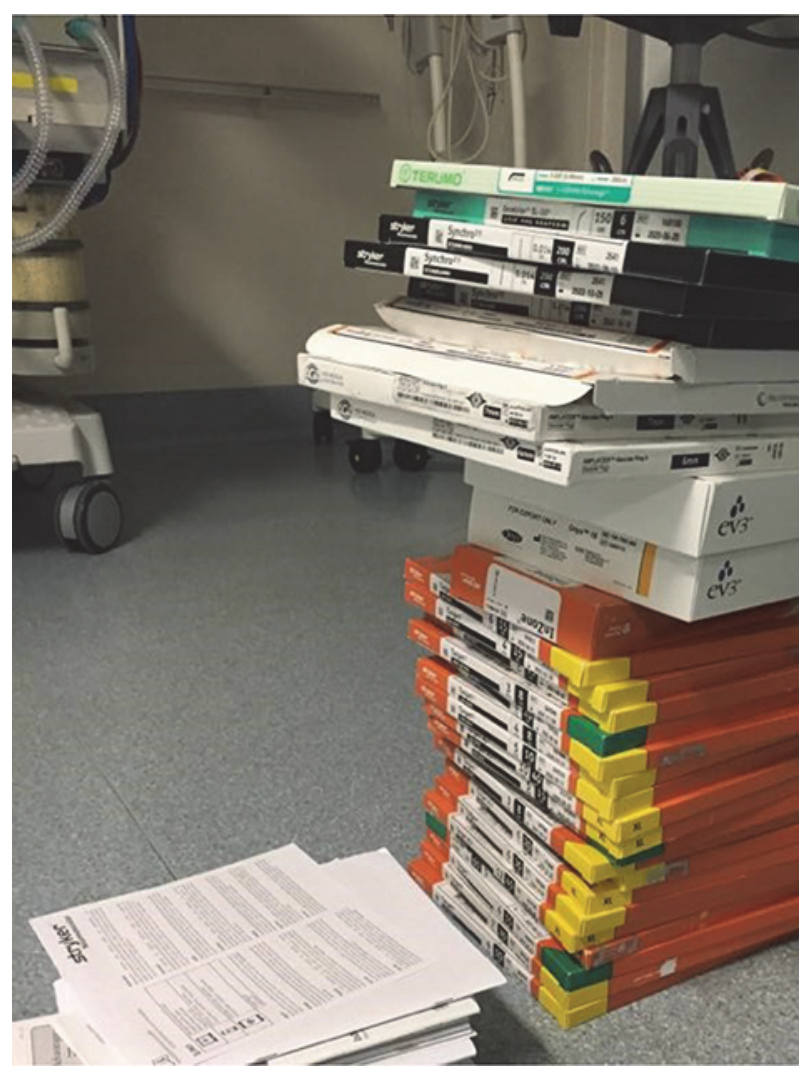

Abstract E-129 Figure 1

appropriate waste segregation have the potential to reduce the environmental impact of our specialty.

Disclosures P. Shum: None. H. Kok: 2; C; Northern Health. J. Maingard: None. M. Schembri: None. M. Banez: None. V. Van Damme: None. C. Barras: 2; C; Royal Adelaide Hospital. L. Slater: 2; C; Monash Health. W. Chong: 2; C; Monash Health. R. Chandra: 2; C; Monash Health. A. Jhamb: 2; C; St Vincent's Hospital. D. Brooks: 2; C; Austin Health. H. Asadi: 2; C; Austin Health, Monash Health.

\section{E-130 STEP FORWARD: EARLY EXPERIENCE USING OCT TECHNOLOGY IN NEUROVASCULAR FIELD}

J Mejia*, J Gutierrez, M Patino Hoyos, V Torres, B Pabon Guerrero. Neurointevencionismo, AngiTeam, Medellin, Colombia

\subsection{6/neurintsurg-2020-SNIS. 162}

Introduction Optical Coherence Tomography (OCT) is a validated technology in cardiac and peripheral vasculature. However, it uses for neurovascular procedures only have been described in few publications of animal lab, cadaveric models and some case reports. Detection of high-resolution (micronscale) findings intra-procedural and during follow-up in the treatment of variety of Neurovascular conditions are described bellow.

Methods Since introduction of this technology in our institution 2018, after learning curve was acquired and IRB approved INR application, we retrospectively described the OCT use to evaluate Intra and extra cranially device implanted. Clinical, anatomical, angiographic variables were registered. Devices such as monolayer and dual-layer flow diverter stents, monolayer and dual-layer carotid stents, and endosaccular flow disruption devices such as the WEB device, were evaluated using this technology.

Results A total of 6 patients were selected to be evaluated using OCT. Eleven Dragonfly Otis Imaging Catheters (Abbott Vascular), assisted with the use of a triaxial system as support were registered. All cases performed under GA and full heparinization. Informed consent obtained. OCT evaluation was performed immediately after the procedure, and in 3 and 6 months as follow-up. Description of multiple endovascular findings in the short and mid-term follow-up were determined, such as wall- apposition, device conformability, presence or not of thrombus device-related, atheroma plaque fragmentation or stabilization, fibrin bridges formation processes, neoendothelization and healing process. Additional information was obtained: aneurismal neck permeability, patency of perforators or arterial ostia, which were clearly elucidated in a close-by histologic scale. In the analysis of the WEB device, none evidence of thrombosis, even none material at the radiopaque-proximal marker, findings in the proximal recess, were revealed. No procedural related complications were reported.

Conclusion Intravascular OCT technology may be suitable to use in Neurovascular procedures highly selected. In our experience an accurate training and learning curve are mandatory. Under special circumstances the use of OCT for INR showed to be feasible and safe. The valuable information acquired still is under analysis but constitutes an extra tool to evaluate intraprocedural results and to assess the healing process during the follow up.

Disclosures J. Mejia: None. J. Gutierrez: None. M. Patino Hoyos: None. V. Torres: None. B. Pabon Guerrero: None.

\section{E-131 A TIME RESOLVED 3D DSA PROTOCOL TO EVALUATE ANEURYSMAL FLOW FOLLOWING FLOW DIVERSION}

${ }^{1} \mathrm{~N}$ Khan*, ${ }^{1,2} \mathrm{~J}$ DiNitto, ${ }^{3} \mathrm{~A}$ Birkhold, ${ }^{1} \mathrm{~A}$ Arthur, ${ }^{1} \mathrm{C}$ Nickele, ${ }^{1} \mathrm{D}$ Hoit, ${ }^{1} \mathrm{~V}$ Inoa, ${ }^{1} \mathrm{~L}$ Elijovich. ${ }^{1}$ Neurosurgery, UTHSC, Memphis, TN; ${ }^{2}$ Siemens Medical Solutions, Malvern, PA; ${ }^{3}$ Neurosurgery, Siemens Healthineers, Forchheim, Germany

\subsection{6/neurintsurg-2020-SNIS.163}

Introduction Time resolved three-dimensional angiography (4D DSA) was developed initially to investigate parameters related to the flow in arterio-venous malformations (AVMs). We have extended this protocol to evaluate flow before and after placement of a flow diverter for treatment of an intracranial aneurysm. Here we evaluate a prototype for 3D iFlow using 4D DSA imaging.

Methods In this study, 4D DSA angiographic imaging (6 s syngo Dyna4D, Siemens Healthineers AG, Forchheim, Germany) was performed on 13 patients before and after receiving a pipeline (Medtronic, USA) across a known intracranial aneurysm. 4D DSA imaging was acquired before a pipeline was placed and after deployment of a pipeline on the same day using a constant flow rate of $3 \mathrm{ml} / \mathrm{sec}$ and $21 \mathrm{cc}$ of contrast. 3D iFlow information was extracted using prototype software from the imaging and 3 bolus arrival time definitions were evaluated.

Results Using three separate reconstruction protocols for $3 \mathrm{D}$ iFlow the flow proximal to the aneurysm and distal to the aneurysm showed similar average bolus arrival times (table 1). 
Abstract E-131 Table 1 Differences in Bolus arrival times after Pipeline placement and before Pipeline placement using two points (one just distal to the aneurysm being treated and one just proximal to the aneurysm being treated)

\begin{tabular}{llllll}
\hline RECON 1 & RECON 1 & RECON & RECON & RECON & RECON \\
Before & After & 2Before & 2After & 3Before & 3After \\
$0.10 \mathrm{~s}( \pm .13)$ & $0.09 \mathrm{~s}( \pm 0.56)$ & $0.08 \mathrm{~s}( \pm 0.13)$ & $0.09 \mathrm{~s}( \pm 0.13)$ & $0.14 \mathrm{~s}( \pm 0.20)$ & $0.16 \mathrm{~s}( \pm 0.33)$ \\
\hline
\end{tabular}

Abstract E-131 Table 2 Differences in Bolus arrival time after Pipeline placement and before Pipeline placement using two points (one just distal to the aneurysm being treated and one within the aneurysm being treated)

\begin{tabular}{lccccc}
\hline RECON 1 & RECON & RECON & RECON & RECON & RECON \\
Before & 1After & 2Before & 2After & 3Before & 3After \\
$0.046 \mathrm{~s}( \pm .14)$ & $0.08 \mathrm{~s}( \pm 0.28)$ & $0.10 \mathrm{~s}( \pm 0.31)$ & $0.01 \mathrm{~s}( \pm 0.12)$ & $0.15 \mathrm{~s}( \pm 0.33)$ & $-0.04 \mathrm{~s}( \pm 0.27)$ \\
\hline
\end{tabular}

The bolus arrival time was then measured at a point just distal to the aneurysm being treated and within the aneurysm itself (table 2). The RECON 1 protocol appeared to most reliably demonstrate slowed contrast inflow into the aneurysm post deployment of the Pipeline device.

Conclusion The 3D iFlow information obtained using 4D DSA imaging can provide clinicians information on the amount of flow pre and post deployment of a pipeline device for treatment of an intracranial aneurysm. This information could be useful to model aneurysm treatment and time to obliteration when utilizing pipeline flow diversion for the treatment of intracranial aneurysms. Continued work to refine protocols and software are needed to implement into clinical care.

Disclaimer This product is under development and not commercially available. Its future availability cannot be ensured.

Disclosures N. Khan: None. J. DiNitto: 5; C; Siemens. A. Birkhold: 5; C; Siemens. A. Arthur: None. C. Nickele: None. D. Hoit: None. V. Inoa: None. L. Elijovich: None.

\section{E-132 MICROPHONE ENABLED CATHETER TO QUANTIFY VENOUS PULSATILE TINNITUS IN PATIENT-SPECIFIC FLOW MODELS}

M Amans*, K Valluru, S Kondapavulur, B Kilbride, H Haraldsson, W Smith, K Meisel, D Saloner. Radiology and Biomedical Imaging, University of California San Francisco, San Francisco, CA

\subsection{6/neurintsurg-2020-SNIS. 164}

Purpose Pulsatile tinnitus (PT) is a debilitating condition that can be caused by abnormal blood flow in venous vasculature near the cochlea ${ }^{1}$. Often times PT is very difficult to diagnose reliably with diagnosis rates typically hovering around $50 \%$ and requiring multiple advanced imaging procedures including diagnostic cerebral angiography ${ }^{2}$. A tool that could objectively mimic a PT patient's hearing experience would allow physicians to reliably diagnose the cause of this difficult symptom.

Materials and Methods We developed a prototype of the 'Phonocatheter', a 6 Fr catheter that can measure and record PT sounds as well as replay them in real time. The Phonocatheter consists of an embedded microphone at its distal end to record intravascular sounds and relay them to a microcontroller (Arduino Mega 2560 Rev3) based custom data acquisition interface. Two benchtop PT flow models ${ }^{3}$ that represent a PT patient's transverse sinus (TS) anatomy before and after lumbar puncture (LP) were created and used to test the Phonocatheter. Glycerol-water mixture was pumped through these models to mimic blood flow at a mean flow rate of $7.4 \mathrm{cc} / \mathrm{s}$. The Phonocatheter was inserted through a $9 \mathrm{Fr}$ access port and was navigated into the TS stenosis region (figure 1A). A handsfree Bluetooth compatible electronic stethoscope was placed externally over the same TS region to record PT transluminally and validate the sound measurements recorded by Phonocatheter $(n=10)$. The sound measurements acquired by Phonocatheter and stethoscope were saved in .wav file format and were exported to MATLAB for comparative analysis. Variation in peak-to-rms sound amplitude values from the TS in pre-LP and post-LP models was calculated. Wilcoxon rank sum test was used to statistically determine the differences in

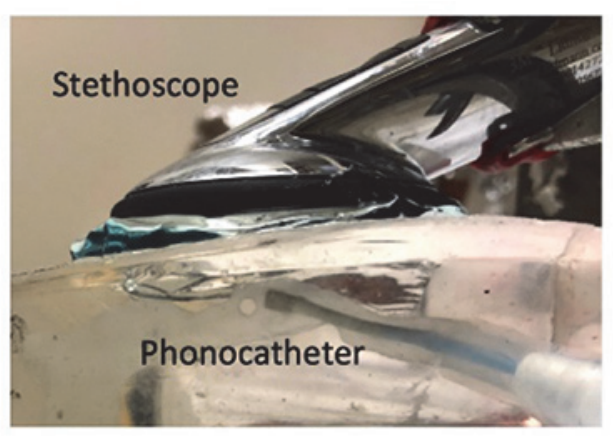

A

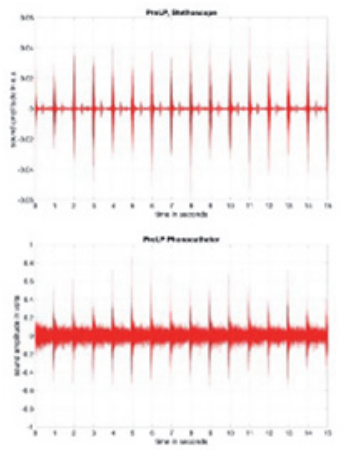

B

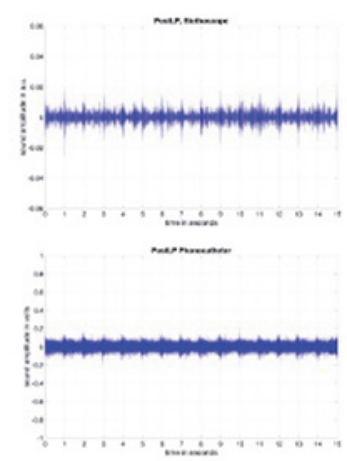

C

Abstract E-132 Figure 1 A) Phonocatheter and a handsfree electronic stethoscope recording sounds generated from transverse sinus stenosis in a patient specific benchtop model of Pulsatile Tinnitus. An external pump (not shown) simulating cardiac rhythm (60 beats per minute) is connected to the flow model to circulate glycerol-water mixture at a characteristic flow rate of $7.4 \mathrm{cc} / \mathrm{s}$. Ultrasound transmission gel was used to couple the stethoscope to the surface of the model. B) Sounds recorded from the model representing the transverse sinus anatomy with stenosis before lumbar puncture (preLP) with stethoscope (top) and phonocatheter (bottom) suggest that the sound was synchronous to the cardiac rhythm mimicking PT. C) Sounds recorded from the model representing the transverse sinus anatomy immediately after lumbar puncture (postLP) with Stethoscope (top) and phonocatheter (bottom) demonstrate that the sound amplitude was weaker compared to the preLP model. There was a reduction in stenosis 